Revue française de la traduction

$215 \mid 2007$

La qualité en perspective

\title{
Traductions externalisées et démarche qualité au Conseil de l'Europe
}

Jean-François Allain

\section{(2) OpenEdition}

Journals

Édition électronique

URL : http://journals.openedition.org/traduire/1274

DOI : 10.4000/traduire.1274

ISSN : 2272-9992

Éditeur

Société française des traducteurs

Édition imprimée

Date de publication : 1 décembre 2007

Pagination : 21-31

ISSN : 0395-773X

\section{Référence électronique}

Jean-François Allain, «Traductions externalisées et démarche qualité au Conseil de l'Europe », Traduire [En ligne], 215 | 2007, mis en ligne le 01 décembre 2007, consulté le 02 mai 2019. URL : http:// journals.openedition.org/traduire/1274; DOI : 10.4000/traduire.1274 


\title{
Traductions externalisées et démarche qualité au Conseil de l'Europe
}

\author{
JeAn-François Allain \\ Chef $d u$ Service de la traduction française \\ Conseil de l'Europe
}

\section{Le contexte}

Le Conseil de l'Europe est une organisation intergouvernementale qui regroupe quarante-sept États mais où $95 \%$ de la communication institutionnelle se fait en anglais et en français, les deux langues officielles. Il est donc doté d'un service de la traduction française et d'un service de la traduction anglaise qui, à eux deux, comptent moins d'une quarantaine de traducteurs et réviseurs. Le service français traduit environ 80000 pages (de 160 mots) par an, dont la moitié est externalisée. En tant que chef de service, j'entretiens donc avec les traducteurs extérieurs des relations de type "donneur d'ordre " à "prestataire de service ». Pour eux, je suis un " client». En réalité, la situation est un peu plus complexe, puisque je suis moi-même prestataire de service pour un autre client (le "service demandeur " dans notre jargon), qui lui-même n'est pas au bout de la chaîne.

Par certains côtés, la situation présente des analogies avec celle d'une agence de traduction, mais la comparaison trouve vite ses limites. Ma position est, en fait, plus proche de celle du maitre d'œuvre (notion française qui ne se traduit pas facilement dans toutes les langues) qui, d'une part coordonne les activités des artisans et sous-traitants à qui il confie ou délègue l'exécution des travaux et, d'autre part, entretient des relations étroites avec le maître d'ouvrage et l'architecte, dont il intègre pleinement les exigences afin de les répercuter au mieux dans la prestation dont il a la responsabilité. 


\section{La problématique}

Un petit exemple illustrera ce terme un peu pompeux mais que je préfere ici à " problème " parce qu'il s'agit d'une chaîne de miniproblèmes qui, ensemble, posent une question de fond. Si je tombe sur une phrase anglaise disant que certains principes (je n'entre pas dans le détail pour « flouter " l'identité de l'organe concerné) « ... are instrumental for a more democratic society and a responsive and accountable governance ", je vois au moins deux mots qui, d'après mon expérience de traducteur, ne sont pas toujours faciles à rendre : instrumental et accountable (la "gouvernance " est aujourd'hui entrée dans les mours). On pourrait en effet se poser des questions sur le poids de instrumental et sur la façon de rendre la notion de accountable. Le traducteur propose « ... qui sont essentielles pour une société plus démocratique ainsi que pour une gouvernance réactive et rendant des comptes".

La phrase n'est sans doute pas du meilleur effet mais, dans ce cas précis, je fais confiance au traducteur, qui s'est sûrement creusé la tête pour trouver la solution la plus appropriée ; je sais aussi que pour ce genre de texte il faut coller à l'original parce que les experts chargés d'adopter le document veulent pouvoir suivre les deux versions en parallèle. Enfin, la phrase vient à la dixième page d'un texte plutôt lourd et confus qui, malgré son importance (c'est une recommandation à notre organe exécutif), ne changera pas la face du monde. Je ne vois donc pas l'intérêt d'intervenir dans le travail du traducteur alors que le texte est réclamé pour le jour même.

Quelques jours plus tard, je reçois un courriel disant : Le projet a été soumis à notre comité [d'experts gouvernementaux] et un mot, entre autres, a donné lieu à beaucoup de débats et explications : responsive que la traduction française a rendu par " réactive ». L'essentiel du débat sur ce mot a été qu'en anglais responsive pouvait englober non seulement des réactions au sens propre du terme mais aussi des actions fondées sur une anticipation. "Proactive " a été proposé un moment, mais en 22 français seulement, surtout pas en anglais, et la décision finale a été de 
demander au service de traduction de retraduire responsive. J'ajoute que le secrétaire francophone du comité se permet de dire que, à son avis, le terme convient, il s'entend répondre : «Mais vous, vous n'êtes pas un gouvernement ! ».

Cette anecdote n'est pas aussi anecdotique qu'il y paraît. Elle montre que nos hommes politiques (et les experts qui les représentent) accordent un poids énorme à des mots qui, pour le commun des mortels, appartiennent à la langue de bois. Surtout, elle met en évidence la primauté du politique sur la linguistique et explique les formulations calamiteuses - résultats de divers marchandages, triturages et autres chipotages - qui caractérisent certains textes internationaux. Enfin, pour ce qui concerne plus directement notre sujet, elle fait apparaître clairement l'existence de toute une chaîne dont il convient maintenant de préciser la nature.

\section{La chaîne de la traduction}

Dans le cas de textes ayant une portée politique, comme l'exemple cité plus haut, il faut distinguer un maître d'ouvrage (les gouvernements des États membres, représentés par leurs ambassadeurs et par les experts qu'ils désignent), l'architecte (le service qui assure le secrétariat des ambassadeurs, d'une commission parlementaire ou d'un comité d'experts), et le maître d'œuvre pour la traduction vers l'autre langue officielle (le service de traduction). À cela, il conviendrait d'ajouter un "utilisateur final " dont la nature est difficile à déterminer (le législateur des pays qui s'engagent à mettre en œuvre les dispositions d'un texte ratifié) et un " destinataire " ou un bénéficiaire qui, souvent, s'ignore en tant que tel, à savoir le citoyen qui bénéficiera de nouvelles dispositions juridiques intégrées dans le droit interne de son pays. La traçabilité de cette chaîne est, on le voit, assez complexe, mais l'exemple extrême du droit de recours individuel, qui permet à toute personne résidant en Europe de saisir la Cour européenne des droits de l'homme pour intenter une action contre l'État dans lequel elle vit, montre que certains textes (et leurs traductions) peuvent prendre pour le citoyen lambda un sens très concret. 
J'aimerais ajouter quelques remarques. D'abord, il est rare - et on peut le regretter - que les traducteurs assistent aux réunions ; il leur est donc difficile d'intégrer les demandes implicites des services demandeurs ou de saisir tous les enjeux politiques d'une formulation linguistique. On pourrait traduire ingénument "minority rights" par "droits des minorités ", mais le délégué d'un pays va affirmer que dans son pays, " il n'y a pas de minorités ! ". Il n'est donc pas question d'octroyer des droits particuliers à des catégories de la population qui n'existent pas. Les interprètes, en revanche, assistent aux discussions mais il est rare qu'ils transmettent aux traducteurs les informations qu'ils peuvent glaner en séance.

Ensuite, le texte traduit ne devient pas un texte autonome, qui aurait coupé le cordon ombilical avec l'original. Peu importe même lequel des deux textes est l'original : les deux versions continuent d'évoluer côte à côte, retouchées par des fonctionnaires et des experts qui travaillent dans une langue qui, dans la majorité des cas, n'est pas leur langue maternelle. Certains connaissent assez les deux langues pour intervenir sur les textes mais pas assez pour le faire à bon escient. Ce genre de discussion révèle au grand jour les problèmes de polysémie et les interprétations connotées : des mots aussi courants que "communauté " ou " citoyenneté " seront historiquement et linguistiquement compris différemment selon les pays et, parfois, les personnes. Il arrive que des débats s'éternisent à la suite de malentendus sur le sens de mots que l'on n'a pas pris suffisamment la peine de définir au préalable.

\section{Le maître d'œuvre}

Dans le schéma que j'ai présenté, le maître d'œuvre est donc le chef du service de la traduction qui décide si les textes doivent être traduits en interne ou en externe. Une externalisation bien pensée permet d'écrêter les pics de la demande et d'améliorer la réactivité, par exemple en jouant sur les week-ends et, parfois, sur les décalages horaires. Cependant, ce n'est pas la préoccupation majeure des financiers, qui 
ont tendance à encourager l'externalisation au seul motif que la page externalisée coûte moins cher que la page traduite en interne. (Ce n'est pas le lieu d'en débattre, mais le surcoût d'un service en interne est en réalité d'environ $30 \%$. C'est le prix à payer pour constituer un capital intellectuel, maintenir une mémoire institutionnelle, assurer la pédagogie nécessaire à l'amélioration constante des savoir-faire et fournir un certain nombre de services non marchands : conseils linguistiques et traductions non rémunérées notamment.)

Dans certaines organisations internationales, le chef de la traduction peut être tenté de confier les traductions externalisées au " premier venu " et de les transmettre aux services demandeurs sans révision pour bien faire comprendre la différence de qualité entre les prestations en interne et les prestations "délocalisées ", et, par conséquent, prouver l'utilité d'un service à demeure. En ce qui nous concerne, la question ne se pose pas, car notre lettre de mission et nos objectifs précisent clairement que la qualité des traductions externalisées doit être de même niveau que celle des traductions internes. Autrement dit, le client ne doit pas savoir si son texte a été traduit en Chine ou dans le bureau d'à côté.

Le premier rôle du maître d'œuvre est donc de vérifier que la prestation fournie par le traducteur à domicile répond aux normes de qualité de la profession. Son second rôle est d'utiliser sa connaissance de l'Organisation et d'intégrer le cahier des charges, souvent implicite, de ses commanditaires pour mettre le texte traduit en conformité avec leurs attentes et avec les usages "maison ». Par exemple, ce n'est pas une faute de traduire " committee " par " comité " mais je ne peux pas laisser passer cette traduction si, en l'occurrence, il s'agit d'une commission.

Dans la réalité, le contrôle qualité exercé par le maître d'œuvre est moins une opération ponctuelle qu'une constellation de tâches, dont certaines interviennent très en amont de la réception du texte. C'est pourquoi je préfere parler de "démarche qualité ». 


\section{Les tâches du maître d'œuvre}

Je me contenterai d'une simple énumération, brièvement commentée, des différentes composantes de cette démarche qualité.

Recruter. La tâche parait simple tant il y a de candidatures spontanées, mais il faut trier! Nous ne faisons pas de test stéréotypé mais confions parfois une première traduction de nature générale. Parfois seulement, car il est délicat de laisser supposer que la collaboration pourra se poursuivre alors que l'on dispose déjà d'un noyau dur de traducteurs compétents qui répondent à la plupart de nos besoins. Les autres voies de recrutement sont les recommandations de traducteurs avec lesquels nous collaborons, et les stages proposés à des étudiants en traduction sélectionnés. Nous essayons d'avoir constamment quelques jeunes (prometteurs) que nous aidons à démarrer dans la profession et que nous formons autant que possible, mais la pédagogie est lourde en temps et il nous est indispensable de disposer aussi d'un nombre suffisant de traducteurs pleinement opérationnels.

Évaluer le niveau général. La première évaluation ne se fait évidemment pas sur des sujets trop techniques ou trop " maison " mais sur les compétences traductionnelles de base : présentation, respect des délais mais, surtout, compréhension de l'original, résistance aux calques, intelligence des situations, facultés de conceptualisation, qualités rédactionnelles. On serait surpris de voir le nombre de personnes qui se prétendent traducteurs et proposent spontanément leurs services avec des $\mathrm{CV}$ qui peuvent faire illusion. Il est évident qu'on ne peut considérer comme professionnel quelqu'un qui écrit : «Dans le domaine des politiques des médias de masse, une nouvelle initiative principale concernera un forum adressant les droits et les responsabilités des médias et les conditions de travail des journalistes ». Si le reste du texte est à l'avenant, on peut écarter d'emblée ce candidat.

Apprécier les forces et les faiblesses. Dès lors qu'un traducteur est retenu comme " espoir ", nous lui fournissons une aide logistique (glossaires, etc.) et nous apprenons à découvrir ses points forts et ses 26 points faibles : certains traduisent bien des originaux bien écrits mais 
sont perdus dès que l'original est mal écrit ou mal pensé ; d'autres ont de bonnes qualités rédactionnelles, appréciables pour traduire les discours ou certaines publications, d'autres ont des compétences juridiques, d'autres enfin sont très rapides ou très disponibles et peuvent dépanner dans des délais très courts tout en assurant une qualité suffisante. Cette connaissance fine des traducteurs est utile pour assurer la meilleure répartition possible des textes et éviter les erreurs de casting. Elle permet de réduire la part d'investissement en contrôle qualité lors du retour du texte.

Apprécier l'enjeu du texte à traduire. Il est d'usage de distinguer les documents de travail des textes destinés à publication. Ils seront traités différemment, du moins si les délais le permettent. Par ailleurs, certains textes apparemment anodins peuvent être très importants par leur destination ; ils ne peuvent donc être confiés à des débutants ou à des traducteurs qui connaissent mal nos activités. Cette analyse préalable du texte doit naturellement prendre en compte les attentes implicites ou explicites des services.

Choisir la bonne adéquation texte-traducteur. Ce choix découle des critères précédents. Il y a les sprinters et les coureurs de fond (qui aiment les très longs textes), les techniciens et les généralistes, les stylistes, les dilettantes qui ne font pas les recherches nécessaires, les débrouillards qui trouvent tout, les méticuleux dont la présentation est toujours impeccable, etc.

Mettre les outils à disposition. Tout nouveau traducteur à domicile reçoit un " kit " : glossaires maison et autres documents terminologiques, liens utiles, etc. De plus, les traductions sont accompagnées de documents de référence, certaines sont prétraitées par la TAO, les citations de textes sont souvent fournies à l'avance. Enfin, le service de terminologie répond aux questions ponctuelles sur les difficultés de traduction.

Effectuer le contrôle qualité proprement dit au moment du retour du texte. Ce contrôle obéit à un savant dosage entre les qualités du traducteur, l'urgence des délais, la destination du texte. Il s'agit donc 
d'un traitement très « différentiel " qui répond à deux fonctions, l'une immédiate - vérifier que le texte est d'une qualité "suffisante " (le concept peut paraître vague, mais pas plus que celui de délai « raisonnable " ou de peine "proportionnée " en droit) et, dans la négative, prendre les mesures correctives appropriées -, l'autre, à plus long terme : apprécier les qualités du traducteur pour mieux le connaître. Selon le cas, le texte reçu est contrôlé par le chef de service ou transmis à un réviseur spécialisé dans tel ou tel secteur d'activité. Les " nouveaux " donnent lieu systématiquement à l'établissement d'une fiche de contrôle qualité, ce qui, après quelques textes, permet de croiser les appréciations. Même un "survol " de la traduction, avec échantillonnage aléatoire et lecture plus attentive des parties les plus importantes (introduction, conclusion, recommandations), peut suffire à déceler des anomalies et à rehausser significativement la qualité du produit (notamment par une bonne utilisation des fonctions chercher/remplacer). L'amélioration peut aussi porter « bêtement " sur la correction d'orthographe (d'excellents traducteurs semblent ignorer cette fonction !) ou sur le formatage (par les secrétaires) de textes difficiles à présenter.

Donner des informations en retour. Le temps ne permet pas de faire des rapports circonstanciés sur chaque texte mais certaines remarques sont consignées sur les fiches de contrôle et, si l'on constate des fautes récurrentes ou des défauts, un courriel permet de donner des conseils ou des consignes. De plus, nous proposons à certains traducteurs des contrats sur site de quelques semaines, qui leur permettent de travailler avec des collègues permanents, d'avoir en direct les conseils des réviseurs, de mieux comprendre le fonctionnement de l'Organisation, etc. Cette "formation par alternance " est très fructueuse pour les deux parties. Elle est facteur de fidélisation, de motivation et d'amélioration des compétences.

Assurer le suivi administratif. Il est certain que le suivi administratif et financier est, pour le traducteur à domicile, un aspect important. Le contrat, établi sur la base d'un comptage départ (sauf pour les rares textes qui n'existent pas en version électronique), est immédiatement 
envoyé au traducteur. $S^{\prime}$ il le faut, nous intervenons auprès des services financiers pour veiller à ce qu'il n'y ait pas de retard indu dans le règlement des honoraires.

Autrement dit, la démarche qualité telle qu'elle est définie ici ne consiste pas seulement à contrôler que le travail a été bien fait. Il vise aussi et surtout à établir une relation bilatérale aussi agréable et équilibrée que possible pour tout le monde.

\section{Et en interne?}

Pour qu'il y ait homogénéité de la prestation de l'ensemble du service, il faut naturellement veiller à ce que la qualité en interne soit elle aussi homogène et d'un niveau au moins égal à celle de la traduction externalisée. De ce point de vue, la démarche qualité prend plusieurs formes : système classique de révision (correction du "produit ", c'està-dire de la traduction terminée, par un réviseur), intervention en cours de processus (accompagnement par des collègues plus expérimentés, ou spécialisés dans certains domaines), relecture croisée entre collègues, mini-formations en interne (par exemple aux nouveaux outils de recherche terminologique, de TAO, etc.).

Par ailleurs, l'Organisation ayant mis en place un système d'appréciation annuelle des agents, les traducteurs doivent - c'est l'un de leurs objectifs - fournir un travail de qualité. Chaque année, ils sont donc invités à fournir plusieurs textes représentatifs de leur production, qui sont évalués par leur appréciateur. Ce système permet de déceler les défauts, les points faibles ou, inversement, les qualités particulières de chacun et de suivre son évolution au fil des années.

Ces quelques remarques entendent montrer que, si l'on veut garantir l'homogénéité de la qualité des traductions, on ne peut avoir deux catégories de traducteurs : une (les permanents) censée bien traduire, l'autre (les traducteurs extérieurs), qu'il faut "surveiller ». Il est certain que les premiers ont une plus grande expérience du fonctionnement 
de l'Organisation et qu'ils peuvent progresser plus facilement par échanges entre pairs, mais les nouveaux moyens de communication et une volonté consciente de pédagogie permettent de niveler ces différences.

\section{L'évaluation des résultats}

Le degré de satisfaction des services demandeurs est officiellement évalué selon deux systèmes. Le premier est le taux de réclamations recevables émanant des services demandeurs. On pourrait penser que les collègues n'osent pas se plaindre, mais ce n'est pas le cas : en effet, la traduction fonctionne selon un système de "refacturation " (les services payent les traductions qu'ils demandent) et certaines personnes sont promptes à accompagner leurs remarques d'un "pour le prix que ça coûte ! ". De plus, nous encourageons ce genre de retour d'information qui nous aide à déceler les anomalies, les erreurs de distribution et autres dysfonctionnements.

Selon nos " indicateurs de performance ", le taux de plaintes doit être inférieur à $5 \%$ du nombre de pages traduites. Depuis deux ans, cet indicateur est inférieur à $2 \%$. Par contre, nous avons des mots de remerciement et de félicitations pour les " excellentes traductions". De plus - c'est un témoignage indirect de satisfaction - certains services demandent que leur texte soit confié à tel ou tel traducteur, interne ou externe.

La deuxième source d'information en retour est l'enquête de satisfaction annuelle menée auprès des clients par notre direction générale et qui vient croiser l'indicateur précédent. Il ressort de cette enquête (qui porte sur les prestations du service informatique, de l'agence de voyage, des services techniques, de la direction des ressources humaines) que plus de $95 \%$ des répondants estiment que la qualité des traductions vers le français est " très bonne » ou "plutôt bonne ". 


\section{En conclusion}

L'expérience que nous menons depuis quelques années dans le service de la traduction française montre que la qualité de la prestation ne peut se réduire à une simple étape de contrôle qui interviendrait à un moment $t$ dans une chaîne de production. C'est un processus à multiples facettes dont le chef de service assure la coordination mais auquel tous les traducteurs permanents participent à des degrés divers. Toutefois, il est important de préciser que la mise en œuvre d'une telle démarche qualité n'est possible que s'il y a personnalisation des rapports avec les prestataires extérieurs, c'est-à-dire si le chef de service dispose d'une latitude suffisante pour recruter les prestataires extérieurs et répartir les textes, ce qui n'est pas le cas dans certaines organisations internationales, où ces tâches sont assurées par un service indépendant. 